\title{
First-in-human, Pharmacokinetic and Pharmacodynamic Phase I Study of Resminostat, an Oral Histone Deacetylase Inhibitor, in Patients with Advanced Solid Tumors
}

\author{
André T. Brunetto ${ }^{1,{ }^{*}}$, Joo Ern Ang ${ }^{1,{ }^{*}}$, Rohit Lal ${ }^{1}$, David Olmos ${ }^{1}$, L. Rhoda Molife ${ }^{1}$, Rebecca \\ Kristeleit $^{1}$, Ann Parker ${ }^{1}$, Isabel Casamayor ${ }^{1}$, Muyibat Olaleye ${ }^{1}$, Anna Mais ${ }^{2}$, Bernhard \\ Hauns $^{2}$, Vera Strobel ${ }^{2}$, Bernd Hentsch ${ }^{2}$, and Johann S. de Bono ${ }^{1}$ \\ ${ }^{1}$ Drug Development Unit, Divisions of Cancer Therapeutics \& Clinical Studies, The Institute of \\ Cancer Research and The Royal Marsden NHS Foundation Trust, Sutton, Surrey, United \\ Kingdom \\ ${ }^{2} 4 \mathrm{SC}$ AG, Planegg-Martinsried, Germany
}

\section{Abstract}

Purpose-This first-in-human dose-escalating trial investigated the safety, tolerability, maximum tolerated dose (MTD), dose-limiting toxicities (DLTs), pharmacokinetics and pharmacodynamics of the novel histone deacetylase (HDAC) inhibitor resminostat in patients with advanced solid tumors.

Experimental Design-Resminostat was administered orally once-daily on days 1-5 every 14 days at 5 dose levels between $100 \mathrm{mg}$ and $800 \mathrm{mg}$. Safety, pharmacokinetics, pharmacodynamics including histone acetylation and HDAC enzyme activity, and antitumor efficacy were assessed.

Results-Nineteen patients (median age 58 years, range 39-70) were treated. At $800 \mathrm{mg}, 1$ patient experienced grade 3 nausea and vomiting, grade 2 liver enzyme elevation, and grade 1 hypokalemia and thrombocytopenia; these were declared as a combined DLT. No other DLT was

Corresponding author: Professor Johann S de Bono, MB ChB, FRCP, MSc, PhD, Professor in Experimental Cancer Medicine, Drug Development Unit, Divisions of Cancer Therapeutics \& Clinical Studies, The Institute of Cancer Research \& The Royal Marsden NHS Foundation Trust, Sutton, Surrey SM2 5PT, United Kingdom, Tel: 44-20-8722-4029, Fax: 44-20-8642-7979, johann.debono@icr.ac.uk.

* André T. Brunetto and Joo Ern Ang contributed equally to this work and are joint first authors.

Presented in part at:

1. AACR - NCI - EORTC 2008, Conference of Molecular Targets and Cancer Therapeutics: An open label, dose escalation trial of oral BYK408740 in patients with advanced malignancy: First-in-man study of a newly developed, oral HDAC inhibitor. J.E. Ang et al

2. HDAC Inhibitors: Targeting Oncology and Beyond, Cambridge Healthtech Conference, 2008: First-in-man phase I trial of BYK408740, an oral HDAC inhibitor, in patients with advanced malignancies J.E. Ang et al

3. ASCO Annual Meeting 2009: A first-in-human Phase I study of 4SC-201, an oral histone deacetylase inhibitor, in patients with solid tumors. A.T. Brunetto et al.

4. ECCO-ESMO Annual Meeting 2009: |Clinical and preclinical development of 4SC-201, a new oral histone deacetylase (HDAC) inhibitor. J.S. de Bono et al.

5. National Cancer Research Institute (NCRI) Conference 2009: A first-in-man, dose-escalating Phase I study of 4SC-201, a new oral histone deacetylase (HDAC) inhibitor in patients with advanced solid tumours. R. Kristeleit et al.

6. HDAC Inhibitors: Targeting Oncology and Beyond, Conference 2009: Preclinical and clinical development of resminostat, a new oral histone deacetylase (HDAC) inhibitor. S.W. Henning et al. 
observed. Although an MTD was not reached and patients were safely dosed up to $800 \mathrm{mg}, 3$ of 7 patients treated with $800 \mathrm{mg}$ underwent dose reductions after the DLT-defining period due to cumulative gastrointestinal toxicities and fatigue. All toxicities resolved following drug cessation. No grade 4 treatment-related adverse event was observed. The pharmacokinetic profile was doseproportional with low inter-patient variability. Pharmacodynamic inhibition of HDAC enzyme was dose-dependent and reached $100 \%$ at doses $\geq 400 \mathrm{mg}$. Eleven heavily pre-treated patients had stable disease and 1 patient with metastatic thymoma had a $27 \%$ reduction in target lesion dimensions.

Conclusions-Resminostat was safely administered with a dose-proportional pharmacokinetic profile, optimal on-target pharmacodynamic activity at dose levels $\geq 400 \mathrm{mg}$ and signs of antitumor efficacy. The recommended phase II dose is $600 \mathrm{mg}$ once-daily on days 1-5 every 14 days.

\section{Keywords}

resminostat; histone deacetylase inhibitor; Phase I trial

\section{INTRODUCTION}

The molecular sequelae of histone deacetylase (HDAC) inhibition are diverse and involve numerous signaling pathways and biological processes that are key to carcinogenesis and cancer progression (1-3). HDAC inhibitors directly cause hyperacetylation of nucleosomal histones leading to tight coiling of chromatin and silencing of expression of genes implicated in the regulation of cell survival, proliferation, differentiation, and apoptosis (4-7). They also impact on other cellular activities, interfering with the recruitment of particular transcription factors to the promoter region of genes that include tumor suppressors (5), and inhibiting heat shock protein 90 (hsp90) by inducing its acetylation with the resultant depletion of hsp90 client proteins including cell cycle proteins (e.g. cdk4), tyrosine kinases (e.g. human epidermal growth factor receptor 2, insulin-like growth factor 1 receptor, MET receptor) and nuclear steroid hormone receptors (e.g. estrogen and androgen receptors) (8-10). HDAC inhibitors are considered promising therapeutic compounds due to their multimodal anticancer effects and potential for combinatorial use with other targeted agents, conventional cytotoxic chemotherapy and radiotherapy $(11,12)$. Multiple compounds in this new class have been in clinical development with vorinostat and romidepsin having gained United States Foods and Drugs Administration (FDA) approval for the treatment of patients with refractory peripheral and cutaneous T-cell lymphoma (13-16).

The hydroxamate HDAC inhibitor, resminostat (formerly 4SC-201, BYK408740), is an oral, potent, pan-inhibitor of class I and II HDAC enzymes. Resminostat inhibits proliferation of a large variety of rodent and human cancer cell lines at sub-to-low micromolar concentrations (Investigator's Brochure, version 5, 2010). Consistent with these findings, dose-dependent induction of histone $\mathrm{H} 3$ hyperacetylation and endogenous $\mathrm{p}^{21 \text { waf1 }}$ were observed in HeLa cells when treated with resminostat. In multiple myeloma cells, proapoptotic signals were seen with increased levels of Bim and Bax, activation of Caspases 3, 8 and 9 and concomitant decrease in levels of Bcl-xL with the application of resminostat (17). Significant antitumor activity was observed after administration in various xenograft tumor models at tolerable oral doses, including the A549 lung cancer model where substantial dose-dependent efficacy of resminostat was demonstrated. In fact, the in vivo preclinical efficacy of resminostat was equal to or higher than that evidenced with currently approved HDAC inhibitors. Additive or synergistic activities of resminostat in combination 
with conventional chemotherapeutic or novel targeted agents were also elicited in these models.

The primary objective of this first-in-human (FIH) study was to investigate the safety and tolerability of repeated oral doses of resminostat using a standard $3+3$ dose-escalation design. Secondary objectives were to identify a dose-schedule for phase II studies, assess pharmacokinetic (PK) and pharmacodynamic (PD) profiles and evaluate anti-cancer activity. PD biomarker assays investigating HDAC enzyme activity and histone acetylation were included to provide proof of on-target activity of resminostat in order to optimally guide the choice of the recommended phase II dose (RP2D).

\section{PATIENTS AND METHODS}

\section{Patient eligibility}

Adult patients with histologically proven advanced solid tumors refractory to standard therapy or for whom no standard therapy existed were eligible. All patients had at least one lesion measurable by response evaluation criteria in solid tumors (RECIST) version 1.0 (18), progressive disease at study entry, Eastern Cooperative Oncology Group performance status (ECOG PS) of 0-1 and expected life expectancy $>3$ months. Acceptable liver, renal and bone marrow function including normal serum electrolytes were mandatory. Patients were required to have recovered from the acute toxicities of any prior therapy and were not allowed any anti-cancer treatment or major surgery in the four weeks preceding trial registration. Patients with clinically significant cardiac or pulmonary disease, symptomatic brain metastasis, active infection, prolonged $\mathrm{QT}_{\mathrm{QT}}$ interval at baseline or previous treatment with an HDAC inhibitor were ineligible.

\section{Trial design and treatment plan}

This first-in-man, open label, dose escalation single-center study was conducted at the Drug Development Unit, The Royal Marsden NHS Foundation Trust, Sutton, United Kingdom, after approval by local institutional review boards and conducted in accordance with Good Clinical Practice guidelines and the Declaration of Helsinki. All patients provided written informed consent before any trial procedure was performed.

The dose of $100 \mathrm{mg}$ administered once-daily on days 1-5 every 14 days was chosen as the recommended safe starting dose in humans based on the no observed adverse effect level (NOAEL) and maximum tolerated dose (MTD) in 5-day rodent and dog species studies. The days 1-5 every 14 day-treatment schedule was chosen based on preclinical data of resminostat and experience with other HDAC inhibitors; the intention was to implement a drug-free period to allow recovery from acute toxicities such as myelosuppression and to limit chronic or cumulative toxicities such as fatigue in order to maximise the administered dose of resminostat. Four dose levels (100 mg, $200 \mathrm{mg}, 400 \mathrm{mg}$ and $800 \mathrm{mg}$ ) were planned; the interim dose level of $600 \mathrm{mg}$ was implemented during the conduct of the study. Intrapatient dose escalation was not permitted.

Resminostat was administered orally (PO) as capsules once-daily (OD) within 30 minutes after a light meal, accompanied by $240 \mathrm{ml}$ of non-carbonated water at room temperature, on days 1-5 every 14 days. Patients fasted for 1 hour post-dose on PK sampling days (1, 5, and 47).

Three to 6 patients were included at each dose level, dependent on the outcome of safety and tolerability evaluations. All patients tolerating the drug were dosed for 4 two-weekly cycles and those showing signs of clinical benefit were given the option of entry into the continuation period during which the use of resminostat was continued in the same way and 
patients were assessed every two-weeks for adverse events until progression of disease or discontinuation from study for any other reason.

A traditional $3+3$ phase I clinical trial design, and standard DLT and MTD criteria were used. Adverse events were graded and reported according to NCI-CTC-AE version 3.0 (19) from commencement of dosing to 30 days after the last administered dose. DLTs were based on toxicities observed in the first 14 days (first cycle). A DLT was defined as: (a) grade 4 neutropenia lasting $>5$ days or associated with fever $>38.5^{\circ} \mathrm{C}$; (b) grade 4 thrombocytopenia; (c) any drug-related non-hematological grade 3 or 4 toxicity with the exceptions of nausea and vomiting, diarrhoea, rash, arthralgia and myalgia if supportive interventions were unsuccessful; and (d) toxicities deemed dose limiting in the opinion of investigators. Fatigue and elevations of GGT were considered dose-limiting if they were of grade 4 severity.

According to the protocol definitions, when a DLT was observed in $\geq 2$ of 3 or 6 patients in any dose cohort, the preceding dose level was declared the MTD provided that the DLT was observed in $\leq 1$ of 6 treated patients treated at that level. A patient was evaluable if he/she received five consecutive daily doses of resminostat and all mandatory safety evaluations were carried out during the nine days following the first dose. Patients who did not meet these minimum study requirements were considered ineligible for the MDT-determining population. Dose adjustments were allowed subsequent to the resolution of toxicities to grade 1 within two weeks of the preceding dose.

\section{Procedures}

Safety evaluations included medical history, physical examination, vital signs, height, weight, review of concomitant medications, and assessment of ECOG performance status (PS). Laboratory studies included urinalysis, full blood count, clotting screen and a comprehensive biochemical and electrolyte panel. A pregnancy test in women of childbearing potential was performed at baseline and prior to each cycle (C).

Patients were assessed at baseline and during the treatment period (C1-4) on days 1 and 2, 5, $8,15,19,29,33,43$ and 47 . For cardiac safety assessments, 12-lead electrocardiographs (ECGs) at rest were carried out pre-dose (within 2 hours), and at $1 \mathrm{~h}, 2 \mathrm{~h}, 3 \mathrm{~h}, 4 \mathrm{~h}, 5 \mathrm{~h}, 6 \mathrm{~h}, 8$ $\mathrm{h}$ and $10 \mathrm{~h}$ post-dose on days 1 and 5. On other visit days, ECG assessments were performed pre-dose and at $1 \mathrm{~h}, 2 \mathrm{~h}, 4 \mathrm{~h}$ and 6-8 h post-dose. In addition, continuous ECG monitoring was performed for up to $24 \mathrm{~h}$ post dose on day 1 for each patient. On day 8, one ECG was recorded ( $72 \mathrm{~h}$ after the last drug administration on day 5) together with a monitoring of concomitant medication and adverse events. Troponin and CK-MB assessments were scheduled at screening and the end-of-study, and at the end of each treatment day during main phase of the trial. They were also carried out if deemed necessary by the investigators.

During the continuation treatment phase, staging was repeated every four treatment cycles (eight weeks). Safety assessments, including vital signs and physical examination, clinical laboratory, ECG, and assessment of adverse events were carried out every 2 to 4 weeks.

\section{PK studies}

Peripheral blood samples for PK analysis were collected on day 1 of $\mathrm{C} 1$ at pre-dose, $15 \mathrm{~min}$, $30 \mathrm{~min}, 45 \mathrm{~min}, 1 \mathrm{~h}, 1.5 \mathrm{~h}, 2 \mathrm{~h}, 2.5 \mathrm{~h}, 3 \mathrm{~h}, 4 \mathrm{~h}, 5 \mathrm{~h}, 6 \mathrm{~h}, 7 \mathrm{~h}, 8 \mathrm{~h}, 9 \mathrm{~h}, 10 \mathrm{~h}$ and $24 \mathrm{~h}$ post-dose. On days 5 and 47 , as patients were not hospitalized, blood was collected at multiple time points from pre-dose up to $8 \mathrm{~h}$ post-dose. Each 2 milliliter $(\mathrm{ml})$ sample was collected in EDTA, placed on ice and centrifuged for $10 \mathrm{~min}$ at $2700 \times \mathrm{g}$ at $4^{\circ} \mathrm{C}$; plasma obtained was stored at $-80^{\circ} \mathrm{C}$ until further analysis. Quantitative analysis of resminostat in the collected 
plasma samples was performed by Nycomed GmbH, Konstanz, Germany, using a validated HPLC-MS/MS method.

\section{PD studies}

Blood samples were taken from all patients for PD studies at the following time points: C1D1 pre-dose and $2 \mathrm{~h}, 5 \mathrm{~h}, 8 \mathrm{~h}$, and $24 \mathrm{~h}$ post-dose; C1D5; and C4D5 (day 47) pre-dose, 2 $\mathrm{h}, 5 \mathrm{~h}$ and $6-8 \mathrm{~h}$ post-dose. On day 8 , one sample was taken from each patient in the $800 \mathrm{mg}$ dose cohort only.

Histone acetylation-Blood samples in citrate were collected for isolation of peripheral blood mononuclear cells (PBMC) in Leucosep tubes. Briefly, erythrocytes were lysed by incubation with Erylysis-Buffer (Qiagen) for 4 minutes, followed by washing with PBS and centrifugation at $400 \mathrm{~g}$ at room temperature for 7 minutes. Cell pellets were then rapidly collected in liquid nitrogen before storage at $-80^{\circ} \mathrm{C}$ until further analysis. The status of histone $\mathrm{H} 4$ acetylation on $\mathrm{N}$-terminal residues was analyzed by Western blotting as follows: PBMCs were first lysed with RIPA buffer, samples with equal protein concentration were then separated using SDS-PAGE followed by the transfer of proteins to a PVDF membrane, and finally, detection of acetylated $\mathrm{H} 4$ histones using specific antibodies (Calbiochem). $\beta$ actin levels were assessed in order to provide a reference for equal protein loading. Signal analysis was performed by densitometric scanning of blots.

Cellular HDAC enzyme activity assay-One $\mathrm{ml}$ aliquots of whole citrate blood were incubated for $2 \mathrm{~h}$ at $37^{\circ} \mathrm{C}$ with the fluorescent HDAC substrate Boc-K(Ac)-AMC. Following erythrocyte lysis, the remaining cells were stored at $-80^{\circ} \mathrm{C}$ until further processing. For determination of HDAC activity, cells were incubated with a developer reagent which led to cell lysis and generation of a fluorescence signal from the deacetylated substrate. The signal was measured by fluorimetry and degree of HDAC activity could thus be calculated (17).

\section{Data analyses}

PK-PK parameter estimates for drug plasma concentration were calculated by noncompartmental analysis using WinNonlin Enterprise, Version 5.2, Pharsight North Carolina (NCA Model 200, extravascular administration). The primary PK variables that were studied included $\mathrm{AUC}_{0-\infty}, \mathrm{C}_{\max }$ and $\mathrm{t}_{1 / 2}$, and these were $\log$-transformed prior to analysis.

Histone acetylation-Densitometric data analysis was performed with AIDA Image Analyser 3.52 software. Acetylated $\mathrm{H} 4$ signal intensities were normalized to $\beta$-actin signals and the relative induction factors (post-dose) were calculated relative to the signal of each untreated patient on day 1 (pre-dose).

Enzyme activity-The measurements in the multi-label counter were expressed as fluorescence units. For analysis of treatment effect, the percentage of enzyme activity/ inhibition was calculated. Here the activity/fluorescent signal with the use of an untreated control was defined as providing $100 \%$ activity while the value obtained after addition of 10 $\mu \mathrm{M}$ trichostatin A (TSA) was defined as $100 \%$ inhibition.

\section{Tumor response}

Computed tomography (CT) and/or magnetic resonant imaging (MRI) scans were carried out every 8 weeks for up to 6 months of resminostat treatment and every 12 weeks thereafter. Response was evaluated using RECIST 1.0 criteria (18). 


\section{RESULTS}

\section{Patient characteristics}

Nineteen patients (10 female, 9 male) received at least one dose of resminostat between September 2007 and January 2009. The median age was 58 years (range 39-70). Twenty six percent of patients were of ECOG PS 0. All patients had confirmed progressive metastatic disease at study entry with a median of 3 sites of metastatic disease (range 2-5) and a median of 2 previous lines of chemotherapy (range 0-6). Baseline patient and tumor characteristics are detailed in Table 1.

\section{Treatment}

A total of 68 treatment cycles were administered to 19 patients at 5 different dose levels (100 mg [n = 3]; $200 \mathrm{mg}$ [n = 3]; $400 \mathrm{mg}$ [n = 3]; $600 \mathrm{mg}$ [n = 3]; $800 \mathrm{mg}$ [n = 7]) during the main study phase. The duration of treatment was consistent across the dose levels, with a median of 4 cycles being administered in all dose levels except $200 \mathrm{mg}$ (median 3, range 2-4).

\section{Safety and tolerability}

Resminostat was generally well-tolerated and the majority of adverse events were mild to moderate in severity. No treatment-related deaths occurred during the study. No patient discontinued or withdrew from the trial due to drug-related adverse events.

DLT/MTD—Eighteen of 19 patients were evaluable for determination of the MTD. One patient with advanced pancreatic carcinoma treated in the $800 \mathrm{mg}$ cohort experienced doselimiting events during the first treatment cycle comprising grade 3 nausea and vomiting despite supportive anti-emetic therapy, in addition to grade 2 alanine transaminase (ALT) increase and grade 1 hypokalemia and thrombocytopenia. No other DLT was reported during the study. At the $800 \mathrm{mg}$ dose level, treatment-related side effects occurring outside of the DLT-defining period, including gastrointestinal toxicities and fatigue, necessitated dose reductions in 3 of 7 patients. For example, a patient with endometrial carcinoma treated at the $800 \mathrm{mg}$ dose level experienced grade 3 hypokalemia secondary to grade 2 nausea and vomiting in cycle 2 and the dose of resminostat was reduced for her in subsequent treatment cycles. Therefore, cumulative chronic fatigue and gastrointestinal toxicities were deemed to be dose limiting at the $800 \mathrm{mg}$ dose level.

Serious adverse events-A total of 3 treatment-related serious adverse events (SAE) were reported in two patients (11\%), both of whom were treated in the $800 \mathrm{mg}$ cohort. One patient required hospitalization in cycle 1 due to grade 3 nausea and vomiting, and again in cycle 2 due to grade 3 elevation of aspartate aminotransferase (AST). Another patient experienced grade 3 hypokalemia secondary to grade 2 nausea and vomiting during treatment in cycle 2 . These adverse events resolved fully following brief drug holidays and the affected patients resumed resminostat treatment at reduced doses. Seven patients (37\%) experienced SAEs that were unrelated to treatment, including 2 patients $(11 \%)$ who died due to progressive disease during the follow-up observation period after discontinuation of resminostat.

Adverse event profile-During the main study phase, i.e. the first 4 treatment cycles, the most frequently reported adverse event, irrespective of causality and grade, was nausea, observed in 41 of 68 administered cycles (60\%); in 40 of these cycles, nausea was grade 1-2 in severity with grade 3 nausea reported in one cycle. The other most frequently reported adverse events included fatigue ( 27 cycles, $40 \%$ ), anorexia ( 21 cycles, $31 \%$ ), dysgeusia (16 
cycles, 24\%) and vomiting (15 cycles, 22\%). Grade 3 fatigue was reported in 3 treatment cycles.

The most common treatment-related adverse events, irrespective of grade, were nausea (13 patients, $68 \%$ ), fatigue ( 8 patients, $42 \%$ ), vomiting ( 7 patients, $37 \%$ ) and anorexia (5 patients, 26\%). In addition, 4 patients (21\%) experienced grade 1-2 dysgeusia. No grade 4 treatment-related adverse event was observed. Table 2 summarizes treatment-related clinical adverse events observed in the main study phase with the exception of laboratory findings which are described below.

Cardiac safety-No drug-related clinical symptom or sign of cardiac impairment was observed. Assessments using 12-lead resting ECGs and continuous telemetric ECG monitoring during day 1 did not reveal any clinically relevant findings. Central review of ECG data detected a moderate dose-dependent increase in heart rate of 20-30 beats per minute. According to central cardiology review, non-specific changes in $\mathrm{T}$ wave morphology such as flattening, notching or slight inversion, were detected at all dose levels except $400 \mathrm{mg}$ (100 mg: 99 ECGs, $200 \mathrm{mg}$ : 99 ECGs, $600 \mathrm{mg}$ : 72 ECGs, and $800 \mathrm{mg}: 105$ ECGs). Discrete ST depression ( $<1 \mathrm{~mm}$ ) was observed at all dose levels except $200 \mathrm{mg}$ (100 mg: 2 ECGs, $400 \mathrm{mg}$ : 40 ECGs, $600 \mathrm{mg}: 3$ ECGs, and $800 \mathrm{mg}: 15$ ECGs). These changes were considered to be clinically insignificant by the investigators and were deemed not to be specifically associated with cardiac ischemia. We note also that transient grade $1 \mathrm{ST}$ depression was observed in 1 patient with markedly increased heart rate (124-159 bpm) treated at the $400 \mathrm{mg}$ dose level; this occurred in the absence of any accompanying symptom or sign, and resolved spontaneously without any intervention. No clear correlation between ST depression and heart rate was observed in all other study patients. Absolute values of $\mathrm{QT}_{\mathrm{c}} \mathrm{B}$ or $\mathrm{QT}_{\mathrm{c}} \mathrm{F}$ intervals $>480 \mathrm{~ms}$ were not detected in central analyses; $\mathrm{QT}_{\mathrm{c}} \mathrm{F}$ values $>450$ $\mathrm{ms}$ were observed in 1 patient only at screening and on C1D1. Another patient developed atrial fibrillation but this was considered unlikely to be drug-related by the investigator as it occurred one week after discontinuation of resminostat treatment and the patient had preexisting arterial hypertension and significant aorto-caval lymphadenopathy. No clinically significant abnormalities in troponin I and CK-MB levels were detected.

Hematological adverse events-Anemia was the most common hematological abnormality and was reported in 17 patients (89\%) regardless of causality; grade 2 and 3 anemia were observed in $9(47 \%)$ and $2(11 \%)$ patients, respectively. Grade 1-2 leukopenia was observed in 7 patients $(37 \%)$ with no grade 3-4 leukopenia reported. Neutropenia was observed in 4 patients $(21 \%)$; one patient experienced grade 3 neutropenia which was considered non-treatment related. No febrile neutropenia or neutropenic infection was reported. Lymphopenia was observed in $89 \%$ of patients and reached grade 2 and 3 in $42 \%$ and $21 \%$ of patients, respectively; grade 4 lymphopenia was not detected. Of note, 10 patients had grade 1 anemia and 11 patients had grade 1-2 lymphopenia at baseline before resminostat treatment. Grade 1-2 thrombocytopenia was seen in 7 patients (37\%) but was not associated with bleeding; there was no evidence of grade 3-4 thrombocytopenia.

Hepatic adverse events-Abnormalities in liver function tests were frequently reported, but were mostly malignancy-related and of grades 1 to 2 in severity. The most frequently reported hepatic abnormality regardless of causality was Y-glutaryltranspeptidase (GGT) elevation; this was reported in 15 patients $(80 \%)$ in total. Grade 3 elevation of GGT was recorded in 2 patients $(11 \%)$ but elevated levels (grades 1 and 3) were already observed at baseline in them. While ALT and AST abnormalities were observed in more than half of the treated patients and reported in $22 \%$ and $21 \%$ of all administered cycles, respectively, elevation of ALT and AST levels were limited to grade 1 and 2 except for 1 patient with grade 3 increase of AST which was deemed to be treatment-related. This AST abnormality 
resolved to baseline levels (grade 1) on a reduced dose of $400 \mathrm{mg}$ of resminostat. No grade 3-4 abnormality in the levels of alkaline phosphatase or total bilirubin was recorded.

Electrolyte imbalances-Abnormal serum electrolyte values were common: hypokalemia and hyponatremia were both reported in 11 patients (58\%) with grade 1 events in $9(47 \%)$ patients and grade 3 events in $2(11 \%)$ patients. Grade 3 hypokalemia was reported in 3 cycles; in both patients, vomiting occurred within 3 days prior to the observation of hypokalemia and was likely to be causative. Grade 3 hyponatremia was reported in 2 cycles; in one instance, this was preceded by vomiting. Hypermagnesemia was reported in 5 patients (26\%) with 1 patient experiencing grade 3 severity. No grade 3 or 4 abnormality in calcium level was observed.

Clotting screen abnormalities-No clinically significant change in the clotting screen was detected except for an ovarian cancer patient with grade 2 deep vein thrombosis (treated with warfarin) and arterial hypertension already present at baseline. In this patient, elevated fibrinogen and partial thromboplastin time and thrombocytopenia were observed prior to the start of treatment cycle 4 . These abnormalities resolved (to normality) following administration of fresh frozen plasma and protamine with continuation of cycle 4 following a one-day delay; these events were deemed not to be treatment-related by the investigators.

Dose delays, modifications and reductions-Of a total of 68 administered cycles, no treatment delay was implemented due to treatment-related adverse event. The duration of treatment was consistent across dose levels with a median of 4 cycles being administered at all dose levels except $200 \mathrm{mg}$ (median 3, range 2-4). Fifty-four cycles (78\%) were administered in their entirety with all 5 doses taken. Of the 19 enrolled patients, 4 (21\%) omitted doses and $4(21 \%)$ underwent 5 dose reductions altogether due to treatment-related AEs. In particular, drug-related fatigue led to dose reductions in 4 patients and an omission in 1 patient; these occurred in 3 patients treated at dose level $800 \mathrm{mg}$ and 1 patient at dose level $600 \mathrm{mg}$.

\section{PK studies}

PK parameters of resminostat were obtained from all dose cohorts and are summarized in Table 3. Observed peak plasma concentration $\mathrm{C}_{\max }$ after single dosing on day 1 was attained between 0.5 to $6 \mathrm{~h}$ (median value at $2 \mathrm{~h}$ ) for all doses. Mean values increased in a dosedependent manner from $487 \mathrm{ng} / \mathrm{mL}$ at the $100 \mathrm{mg}$ dose level to $4,970 \mathrm{ng} / \mathrm{mL}$ at the $800 \mathrm{mg}$ dose level. Terminal plasma elimination half-life on day 1 for all doses was constant within the range of 2.35 and $4.41 \mathrm{~h}$. Plasma exposure $\mathrm{AUC}_{\mathrm{inf}}$ increased proportionally with increasing doses ranging from 1236 to $21,200 \mathrm{hr} \cdot \mathrm{ng} / \mathrm{mL}$ (mean values) after a single dose.

The same pattern of PK characteristics was also observed on day 5 after repeated doses. There was no significant change in both the AUC and $\mathrm{C}_{\max }$ between treatment days 1 and 5 for all studied dose levels. The number of patients with plasma sampling on day 47 was limited $(\mathrm{n}=10)$ but in these patients, comparison of PK characteristics between days 5 and 47 did not reveal any significant difference.

\section{PD studies}

HDAC enzyme activity-The degree of HDAC enzyme inhibition measured by a functional enzyme assay in PBMCs was dose-dependent and ranged from 50\% to $100 \%$. Enzyme inhibition was time-dependent and reversible in all dose groups. Drug plasma levels were related to HDAC enzyme inhibition during dose escalation; statistical correlation of the degree of HDAC inhibition at 2 hour post-dose on day 1 for all dose levels with drug plasma concentrations at the same time point revealed a coefficient of determination, $\mathrm{R}^{2}$, of 0.875 , 
and resminostat concentration at 50\% of HDAC enzyme inhibition of $306 \mathrm{ng} / \mathrm{ml}$ using the Hill model (Supplementary Figure 1) (20). Maximal enzyme inhibition of up to $100 \%$ was detected at dose levels $\geq 400 \mathrm{mg}$ after single dose administration and on days 5 and 47 following repeated dosing (Figure 1a).

Histone acetylation-The median histone $\mathrm{H} 4$ acetylation level increased by at least 4-5 fold after dosing at all studied dose levels; a marked increase (10 fold) was observed at the $800 \mathrm{mg}$ dose level. A high degree of inter-patient variability in hyperacetylation status was observed, particularly in the cohort of patients treated at the $800 \mathrm{mg}$ dose level (Figure 1b).

\section{Recommended phase II dose}

Based on the safety, PK and PD data, $600 \mathrm{mg}$ resminostat administered orally once-daily on days 1-5 every 14 days was declared the RP2D.

\section{Antitumor activity}

A total of 18 patients (95\%) were evaluable for response by RECIST. One patient treated in the $800 \mathrm{mg}$ dose level was not evaluable for response, having discontinued from the study during the first cycle, with no evidence of progressive disease and not undergone any ontreatment efficacy assessment. Eleven patients (58\%, 95\% CI: $34-80)$ had stable disease (SD) lasting $>8$ weeks. Three of 3 patients in the $600 \mathrm{mg}$ dose group and 5 of 6 patients treated on the $800 \mathrm{mg}$ dose level achieved SD; by contrast, 3 of 9 patients treated at the lower dose levels achieved SD. A patient with metastatic thymoma achieved SD as best RECIST-based response but demonstrated a $27 \%$ reduction in the sum of the longest diameters of tumor target lesions and remained on study for 8.7 months (19 cycles) (Figure 2). Another patient with liposarcoma remained on study with SD for 14.2 months (31 cycles), having confirmed disease progression before trial participation.

\section{DISCUSSION}

In this first-in-human study, resminostat was well tolerated at the tested doses and schedule with no unexpected toxicities. Fatigue and gastrointestinal toxicities were cumulative and led to dose reductions at the $800 \mathrm{mg}$ dose level; the formally-defined MTD was not reached with 1 of 6 evaluable patients sustaining a DLT at this dose level. PK was linear across the dose range tested while HDAC enzyme inhibition was satisfactorily achieved at doses $\geq 400$ $\mathrm{mg}$. Based on the combined safety, PK and PD profiles, $600 \mathrm{mg}$ resminostat given oncedaily for 5 days in a 14-day cycle was declared as the RP2D. This well-tolerated doseschedule demonstrated biological activity coupled to signs of clinical efficacy with longterm dosing.

The clinical toxicities of resminostat are consistent with those reported for other HDAC inhibitors (21). Principal treatment-related adverse events included fatigue ( $42 \%$ of patients), and nausea and vomiting (68\% and 37\%, respectively); the latter two symptoms were manageable with the use of standard anti-emetics. In contrast with other known HDAC inhibitors, at the dose range tested, resminostat did not cause clinically significant myelosuppression. Anemia and lymphopenia were the most frequent hematological abnormalities reported (89\% of patients), with no grade 4 events observed. Of note, almost $60 \%$ of patients had anemia or lymphopenia at baseline prior to resminostat treatment. Leucopenia and thrombocytopenia were less common (37\% and 37\%, respectively), and were limited to grade 1 or 2 . Neutropenia was rare but one patient developed grade 3 neutropenia which was considered to be non-treatment related; no febrile neutropenia or neutropenic infection was reported. This profile suggests that the future development of resminostat in combination with myelosuppressive agents will be feasible. 
Prolongation of the $\mathrm{QT}_{\mathrm{c}}$ interval has been reported in patients treated with other HDAC inhibitors $(22,23)$. By contrast, central review of all ECGs recorded in this study revealed no evidence of significant changes in the $\mathrm{QT}_{\mathrm{c}}$ interval, suggesting that resminostat does not impact on the duration of myocardial repolarization at the dose range and schedule tested. A moderate increase of the heart rate associated with drug administration was observed in some patients. At doses $\geq 400 \mathrm{mg}$, non-specific flattening of T-wave and/or slight depression of the ST-segment was seen as previously described with other HDAC inhibitors.

Notwithstanding the absence of significant $\mathrm{QT}_{\mathrm{c}}$ interval prolongation and clinically important cardiac adverse events, the monitoring and prompt correction (if abnormal) of plasma levels of potassium and magnesium are recommended prior to commencement of treatment. Of importance, potential gastrointestinal toxicities including nausea, vomiting, and diarrhea can cause electrolyte imbalance predisposing patients to cardiac rhythm changes (24). These can be difficult to differentiate from direct potential cardiac effects of HDAC inhibitors.

The observed inter- and intra-patient variability of single- and multiple PK dose profiles was low. PK data did not indicate any trend to accumulation as expected (in the context of the dosing regimen) due to the relatively short plasma elimination half-life of approximately 4 h. A dose proportional increase in AUC and $\mathrm{C}_{\max }$ for all dose levels and at different days was observed. There was no change in $t_{1 / 2}$ with dose increments, indicating linear PK within the tested dose range. No time-dependent PK characteristic was observed to suggest the saturation or inhibition of any clinically relevant elimination mechanism. The high plasma exposure observed indicates good oral bioavailability in this setting.

PD assessments comprising functional assessment of HDAC enzyme inhibition and histone acetylation status provided proof-of-target modulation by resminostat. In the histone $\mathrm{H} 4$ acetylation assay, a median increase of acetylation by at least 5 -fold after resminostat dosing was observed at all dose levels. Although a median 10-fold increase in the degree of histone $\mathrm{H} 4$ acetylation was observed at the highest dose level of $800 \mathrm{mg}$, inter-patient variability was markedly high in this dose cohort. Densitometric quantification of H4 acetylation is, however, relatively insensitive and imprecise. Hence, we additionally incorporated the use of a robust, analytically validated and novel functional enzyme assay of HDAC activity in this study. Using this assay, we observed that HDAC enzyme inhibition was correlated to drug plasma concentrations and that maximal inhibition (i.e. 100\%) of HDAC was achieved at dose levels $\geq 400 \mathrm{mg}$. These PD biomarker readouts crucially augmented confidence in the choice of a RP2D.

Currently, there exists a wide range of potent, structurally diverse HDAC inhibitors. The reasons underlying the distinct toxicity profiles of different HDAC inhibitors are poorly understood but may be due to differences in ADME properties, HDAC isotype inhibition profiles and/or off-target effects $(1,12,21-23)$. Nonetheless, HDAC inhibitors seem to share a common adverse event profile, which includes gastrointestinal disturbances and myelosuppression. In contrast with other HDAC inhibitors, at the studied dose levels, resminostat did not cause significant myelosuppression, which renders it a potentially suitable agent for combinatorial use with myelosuppressive drugs, and the absence of clinically significant cardiac events including $\mathrm{QT}_{\mathrm{c}}$ interval prolongation was also striking. These are in spite of clear evidence of target modulation at the RP2D. Of note, direct comparison of the degree of pharmacodynamic marker modulation by resminostat with other HDAC inhibitors is difficult to make due to lack of published data. Moreover, dissimilar assays used in different clinical trials and the novelty of the HDAC enzyme inhibition assay presented in this study further restrict any comparative analysis. 
In this study, 11 patients (58\%) achieved SD as best response at 8 weeks of treatment (4 cycles). One patient with thymoma demonstrated a marginal response lasting 8.7 months. Of interest, belinostat, an inhibitor of HDAC class I and II enzymes, demonstrated clinical activity in a phase II study of 32 patients with recurrent or metastatic thymoma and thymic carcinoma (25). Evaluation of resminostat as monotherapy in a Phase II clinical trial of relapsed/refractory Hodgkin lymphoma patients after high dose chemotherapy and autologous hematopoietic stem cell transplantation has yielded encouraging results; in a heavily pre-treated group of patients (median of 6 prior lines of therapy), the reported objective response rate by an independent central review board in an interim analysis was $33 \%$ ( 11 of 33 evaluable patients) by the Cheson criteria with a clinical benefit rate of 55\% (18 of 33 patients) $(26,27)$. Resminostat is also being developed in combination with sorafenib in hepatocellular carcinoma (28) and with standard FOLFIRI treatment in colorectal cancer (ClinicalTrials.gov NCT01277406). Overall, emerging tolerability and efficacy data from these ongoing studies are promising and the final results are awaited (27, 28).

In summary, this first-in-human study demonstrated that the novel, hydroxamate, panHDAC inhibitor resminostat is well tolerated and can be safely administered to patients with a RP2D of $600 \mathrm{mg}$ PO OD on days 1-5 every 14 days. Favorable PK properties with satisfactory target modulation were confirmed, and signs of clinical activity were elicited with the use of resminostat as monotherapy.

\section{Supplementary Material}

Refer to Web version on PubMed Central for supplementary material.

\section{Acknowledgments}

Dr. Brunetto was awarded a 2009 ASCO Annual Meeting Merit Award for the presentation of part of this work by the ASCO Foundation. This study was supported up to 31 March 2009 by Nycomed GmbH, Byk-Gulden-Str. 2, 78467 Konstanz (Germany); and from 1 April 2009, by 4SC AG, Am Klopferspitz 19a, 82152 Planegg-Martinsried (Germany). The Drug Development Unit of The Royal Marsden NHS Foundation Trust and The Institute of Cancer Research was supported in part by a program grant from Cancer Research U.K. Support was also provided by the Experimental Cancer Medicine Center (to The Institute of Cancer Research), the National Institute for Health Research Biomedical Research Center (jointly to The Royal Marsden NHS Foundation Trust and The Institute of Cancer Research) and the Wellcome Trust (grant 090952/Z/09/Z to Dr. Ang).

\section{REFERENCES}

1. Ropero S, Esteller M. The role of histone deacetylases (HDACs) in human cancer. Mol Oncol. 2007; 1:19-25. [PubMed: 19383284]

2. Bolden JE, Peart MJ, Johnstone RW. Anticancer activities of histone deacetylase inhibitors. Nat Rev Drug Discov. 2006; 5:769-84. [PubMed: 16955068]

3. Gray SG, Qian CN, Furge K, Guo X, Teh BT. Microarray profiling of the effects of histone deacetylase inhibitors on gene expression in cancer cell lines. Int J Oncol. 2004; 24:773-95. [PubMed: 15010814]

4. Workman JL, Kingston RE. Alteration of nucleosome structure as a mechanism of transcriptional regulation. Annu Rev Biochem. 1998; 67:545-79. [PubMed: 9759497]

5. Arts GJ, Langemeijer E, Tissingh R, Ma L, Pavliska H, Dokic K, et al. Adenoviral vectors expressing siRNAs for discovery and validation of gene function. Genome Res. 2003; 13:2325-32. [PubMed: 12975310]

6. Jones PA, Baylin SB. The fundamental role of epigenetic events in cancer. Nat Rev Genet. 2002; 3:415-28. [PubMed: 12042769] 
7. Richon VM, Sandhoff TW, Rifkind RA, Marks PA. Histone deacetylase inhibitor selectively induces p21WAF1 expression and gene-associated histone acetylation. Proc Natl Acad Sci U S A. 2000; 97:10014-9. [PubMed: 10954755]

8. George P, Bali P, Annavarapu S, Scuto A, Fiskus W, Guo F, et al. Combination of the histone deacetylase inhibitor LBH589 and the hsp90 inhibitor 17-AAG is highly active against human CML-BC cells and AML cells with activating mutation of FLT-3. Blood. 2005; 105:1768-76. [PubMed: 15514006]

9. Rahmani M, Reese E, Dai Y, Bauer C, Kramer LB, Huang M, et al. Cotreatment with suberanoylanilide hydroxamic acid and 17-allylamino 17-demethoxygeldanamycin synergistically induces apoptosis in Bcr-Abl+ Cells sensitive and resistant to STI571 (imatinib mesylate) in association with down-regulation of Bcr-Abl, abrogation of signal transducer and activator of transcription 5 activity, and Bax conformational change. Mol Pharmacol. 2005; 67:1166-76. [PubMed: 15625278]

10. Kovacs JJ, Murphy PJ, Gaillard S, Zhao X, Wu JT, Nicchitta CV, et al. HDAC6 regulates Hsp90 acetylation and chaperone-dependent activation of glucocorticoid receptor. Mol Cell. 2005; 18:601-7. [PubMed: 15916966]

11. Karagiannis TC, El-Osta A. Will broad-spectrum histone deacetylase inhibitors be superseded by more specific compounds? Leukemia. 2007; 21:61-5. [PubMed: 17109024]

12. Glaser KB, Staver MJ, Waring JF, Stender J, Ulrich RG, Davidsen SK. Gene expression profiling of multiple histone deacetylase (HDAC) inhibitors: defining a common gene set produced by HDAC inhibition in T24 and MDA carcinoma cell lines. Mol Cancer Ther. 2003; 2:151-63. [PubMed: 12589032]

13. Piekarz RL, Frye R, Prince HM, Kirschbaum MH, Zain J, Allen SL, et al. Phase 2 trial of romidepsin in patients with peripheral T-cell lymphoma. Blood. 2011; 117:5827-34. [PubMed: 21355097]

14. Piekarz RL, Frye R, Turner M, Wright JJ, Allen SL, Kirschbaum MH, et al. Phase II multiinstitutional trial of the histone deacetylase inhibitor romidepsin as monotherapy for patients with cutaneous T-cell lymphoma. J Clin Oncol. 2009; 27:5410-7. [PubMed: 19826128]

15. Mann BS, Johnson JR, Cohen MH, Justice R, Pazdur R. FDA approval summary: vorinostat for treatment of advanced primary cutaneous T-cell lymphoma. Oncologist. 2007; 12:1247-52. [PubMed: 17962618]

16. Mann BS, Johnson JR, He K, Sridhara R, Abraham S, Booth BP, et al. Vorinostat for treatment of cutaneous manifestations of advanced primary cutaneous T-cell lymphoma. Clin Cancer Res. 2007; 13:2318-22. [PubMed: 17438089]

17. Mandl-Weber S, Meinel FG, Jankowsky R, Oduncu F, Schmidmaier R, Baumann P. The novel inhibitor of histone deacetylase resminostat (RAS2410) inhibits proliferation and induces apoptosis in multiple myeloma (MM) cells. Br J Haematol. 2010; 149:518-28. [PubMed: 20201941]

18. Therasse P, Arbuck SG, Eisenhauer EA, Wanders J, Kaplan RS, Rubinstein L, et al. New guidelines to evaluate the response to treatment in solid tumors. European Organization for Research and Treatment of Cancer, National Cancer Institute of the United States, National Cancer Institute of Canada. J Natl Cancer Inst. 2000; 92:205-16. [PubMed: 10655437]

19. Program CTE. Common Terminology Criteria for Adverse Events. 2006

20. Hill AV. The possible effects of the aggregation of the molecules of hæmoglobin on its dissociation curves. The Journal of physiology. 1910; 40:iv-vii.

21. Prince HM, Bishton MJ, Harrison SJ. Clinical studies of histone deacetylase inhibitors. Clin Cancer Res. 2009; 15:3958-69. [PubMed: 19509172]

22. Lane AA, Chabner BA. Histone deacetylase inhibitors in cancer therapy. J Clin Oncol. 2009; 27:5459-68. [PubMed: 19826124]

23. Marsoni S, Damia G, Camboni G. A work in progress: the clinical development of histone deacetylase inhibitors. Epigenetics. 2008; 3:164-71. [PubMed: 18487953]

24. Molife R, Fong P, Scurr M, Judson I, Kaye S, de Bono J. HDAC inhibitors and cardiac safety. Clin Cancer Res. 2007; 13:1068. author reply -9. [PubMed: 17289905] 
25. Giaccone G, Rajan A, Berman A, Kelly RJ, Szabo E, Lopez-Chavez A, et al. Phase II study of belinostat in patients with recurrent or refractory advanced thymic epithelial tumors. J Clin Oncol. 2011; 29:2052-9. [PubMed: 21502553]

26. Cheson BD, Pfistner B, Juweid ME, Gascoyne RD, Specht L, Horning SJ, et al. Revised response criteria for malignant lymphoma. J Clin Oncol. 2007; 25:579-86. [PubMed: 17242396]

27. Walewski J, Paskiewicz-Kozik E, Warszewska A. Final Results of the Phase II SAPHIRE Trial of Resminostat (4SC-201) in Patients with Relapsed/Refractory Hodgkin Lymphoma. Blood (ASH Annual Meeting Abstracts). 2011; 118 Abstract 2675.

28. Bitzer M, Horger M, Ebert P, Ganten M, Woerns B, Hauns M, et al. First clinical data of resminostat, a novel oral histone deacetylase (HDAC) inhibitor, in patients with hepatocellular carcinoma (HCC): The SHELTER study. Journal Of Clinical Oncology. Chicago: Journal Of Clinical Oncology. 2010 p. suppl; abstr e14661. 


\section{TRANSLATIONAL RELEVANCE}

In this first-in-human study, the novel oral hydroxamate pan-HDAC inhibitor resminostat was safely administered and well tolerated when used as a single agent at doses associated with therapeutically relevant plasma concentrations, with evidence of antitumor activity. The choice of a recommended phase II dose/schedule was jointly guided by data on safety and pharmacokinetic profiles, coupled to observations of optimal modulation of pharmacodynamic markers including HDAC enzyme inhibition and histone acetylation at the chosen dose level. Resminostat did not show any major, dose-limiting hematological toxicity at the dose levels assessed and has the potential to be used in combination with myelosuppressive anticancer agents. Resminostat is currently being evaluated in Phase II clinical trials in combination with sorafenib in hepatocellular carcinoma, with standard FOLFIRI treatment in colorectal carcinoma, and as monotherapy in relapsed/refractory Hodgkin's lymphoma, with encouraging safety and efficacy profiles. 


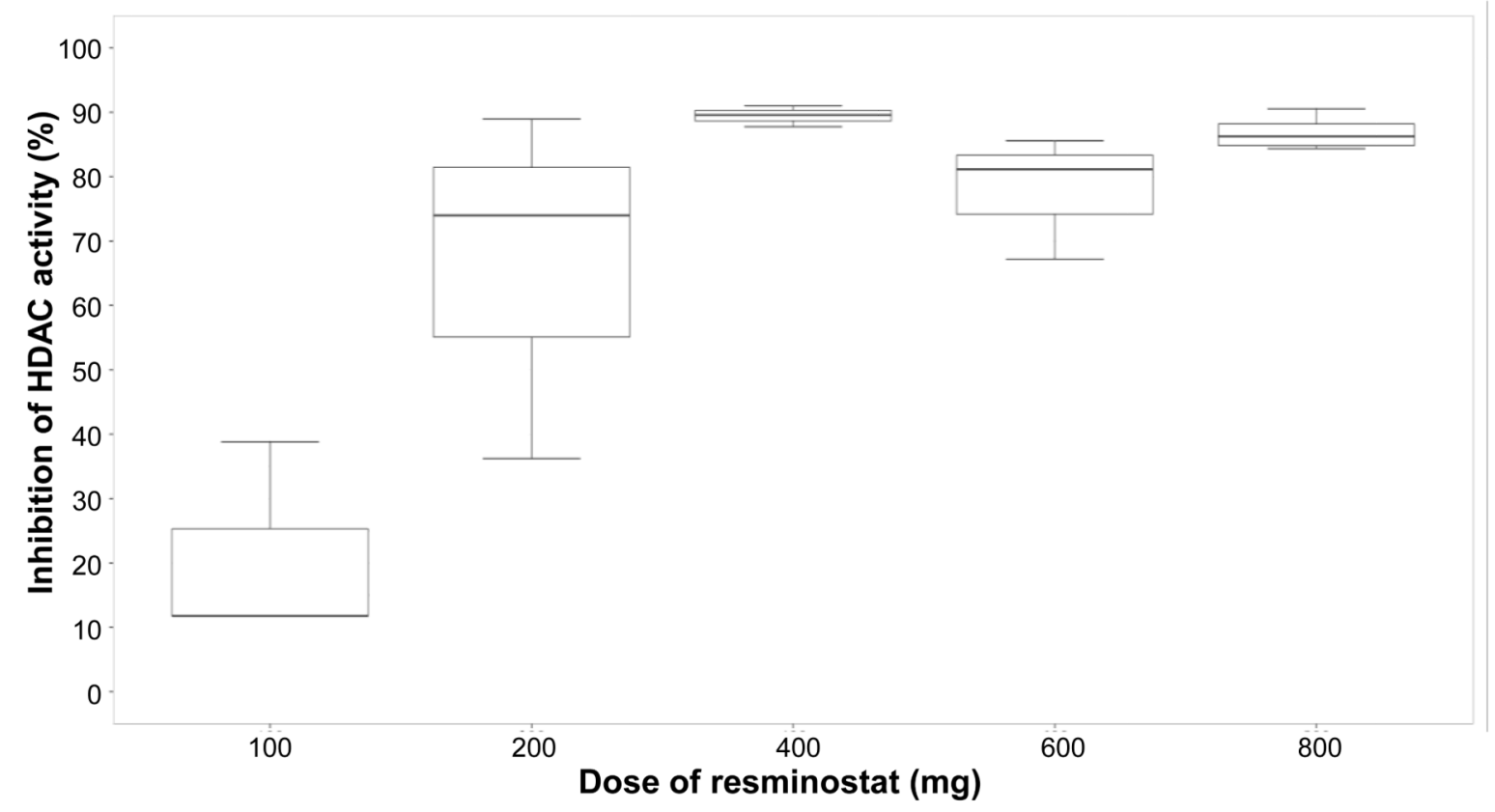

Figure 1a.

Box plots of HDAC enzyme inhibition at 5 hours post-dose in PBMC after single dose of resminostat on C1D1; dose levels $100 \mathrm{mg}$ to $600 \mathrm{mg}$ : $\mathrm{N}=3$ each, $800 \mathrm{mg}$ dose level: $\mathrm{N}=6$. The lower and upper boundaries of each box define the 25th and 75th percentiles; the horizontal line in each box indicates the median; and the bars define the lowest and highest values, excluding outliers. 


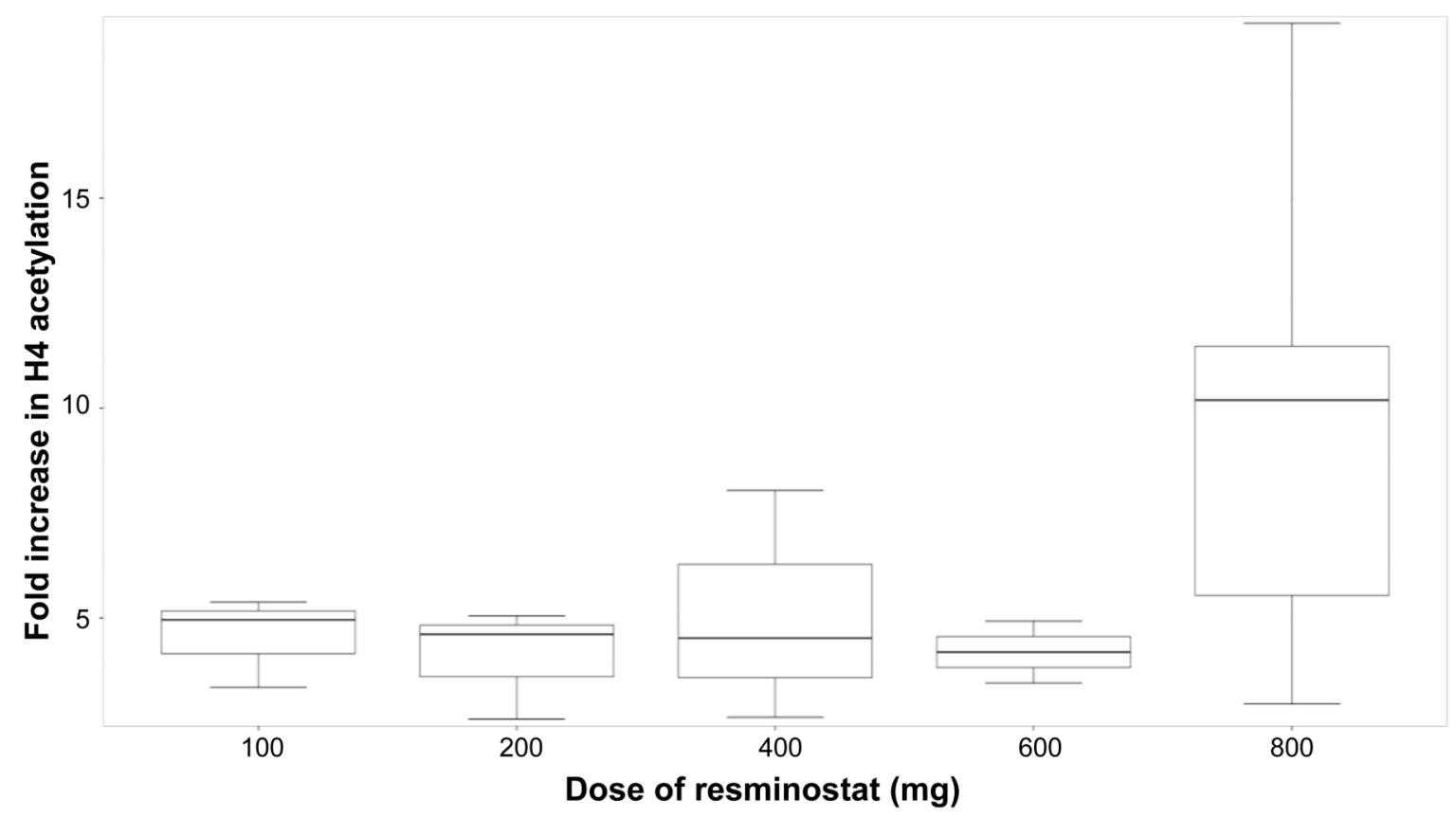

Figure 1b.

Box plots of median fold increase in histone $\mathrm{H} 4$ acetylation in PBMC after single dose of resminostat on C1D1; maximum fold increase of histone acetylation was chosen for each patient. Dose levels $100 \mathrm{mg}$ to $600 \mathrm{mg}$ : N=3 each, $800 \mathrm{mg}$ dose level: $\mathrm{N}=6$. The lower and upper boundaries of each box define the 25th and 75th percentiles; the horizontal line in each box indicates the median; and the bars define the lowest and highest values, excluding outliers. 

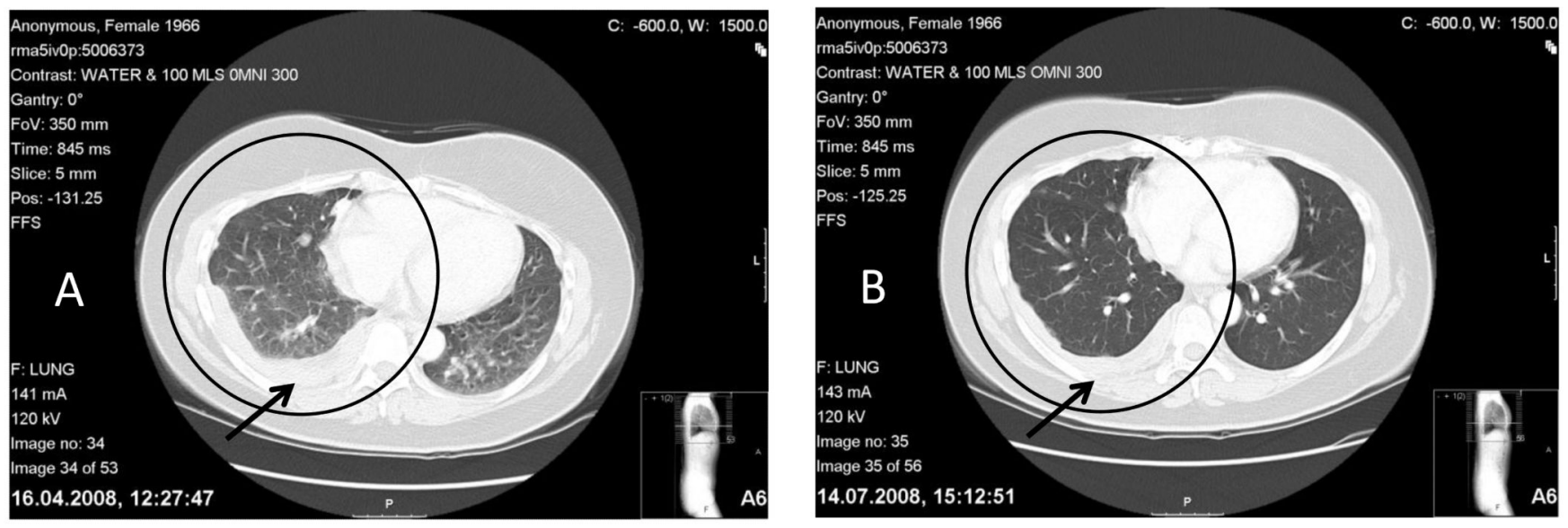

Figure 2.

A 42 year old female patient with mediastinal thymoma (Masaoka stage IV, extensive pleural based deposit pointed to by arrow) who received 3 previous lines of systemic therapy remained on study for 19 cycles with evidence of tumor shrinkage (SD by RECIST). In CTbased assessments, target lesions at baseline (panel A) included a large tumor at the right lung base involving the diaphragmatic pleural surface and a right pulmonary horizontal fissure nodule which reduced in size after 4 treatment cycles (panel B). In addition, the conglomerate of nodular lesions in the right lung decreased in size with a reduction in the degree of pulmonary infiltrates (non-target lesions within encircled regions). 
Table 1

Patient and tumor characteristics

\begin{tabular}{|c|c|c|c|c|c|c|c|}
\hline \multirow[t]{2}{*}{ Dose level } & \multirow{2}{*}{$\frac{100 \mathrm{mg}}{\mathrm{N}=3}$} & \multirow{2}{*}{$\frac{200 \mathrm{mg}}{\mathrm{N}=3}$} & \multirow{2}{*}{$\begin{array}{c}400 \mathrm{mg} \\
\mathrm{N}=3\end{array}$} & \multirow{2}{*}{$\begin{array}{c}600 \mathrm{mg} \\
\mathrm{N}=3\end{array}$} & \multirow{2}{*}{$\begin{array}{c}800 \mathrm{mg} \\
\mathrm{N}=7\end{array}$} & \multicolumn{2}{|c|}{ Total } \\
\hline & & & & & & $N=19$ & $\%$ \\
\hline \multicolumn{8}{|l|}{ Gender } \\
\hline Male & 2 & 3 & 2 & - & 2 & 9 & 47 \\
\hline Female & 1 & - & 1 & 3 & 5 & 10 & 53 \\
\hline \multicolumn{8}{|l|}{ Age (years) } \\
\hline Median (range) & $57(55-61)$ & $58(43-64)$ & $64(62-66)$ & $50(41-62)$ & $49(39-70)$ & $58(39-$ & \\
\hline \multicolumn{8}{|l|}{ ECOG PS } \\
\hline 0 & - & 1 & - & 2 & 2 & 5 & 26 \\
\hline 1 & 3 & 2 & 3 & 1 & 5 & 14 & 74 \\
\hline \multicolumn{8}{|l|}{ Tumor Types } \\
\hline Colorectal & - & 2 & - & - & 1 & 3 & 16 \\
\hline NSCLC & 1 & - & 2 & - & - & 3 & 16 \\
\hline Ovarian & 1 & - & - & 1 & 1 & 3 & 16 \\
\hline Cholangiocarcinoma & - & 1 & - & - & 1 & 2 & 11 \\
\hline Mesothelioma & 1 & - & 1 & - & - & 2 & 11 \\
\hline Endometrial & - & - & - & - & 1 & 1 & 5 \\
\hline Liposarcoma & - & - & - & 1 & - & 1 & 5 \\
\hline Melanoma & - & - & - & - & 1 & 1 & 5 \\
\hline Merkel cell cancer & - & - & - & - & 1 & 1 & 5 \\
\hline Pancreatic & - & - & - & - & 1 & 1 & 5 \\
\hline Thymoma & - & - & - & 1 & & 1 & 5 \\
\hline $\begin{array}{l}\mathrm{N} \text { patients with prior } \\
\text { surgery }\end{array}$ & 3 & 3 & 1 & 3 & 7 & 17 & 89 \\
\hline $\begin{array}{l}\mathrm{N} \text { patients with prior } \\
\text { radiotherapy }\end{array}$ & 2 & 1 & 3 & 1 & 2 & 9 & 47 \\
\hline $\begin{array}{l}\text { N patients with prior } \\
\text { chemotherapy }\end{array}$ & 3 & 3 & 3 & 2 & 6 & 17 & 89 \\
\hline $\begin{array}{l}\text { Median } \mathrm{N} \text { of prior } \\
\text { chemotherapy lines } \\
\text { (range) }\end{array}$ & $2(1-5)$ & $3(2-6)$ & $2(2-6)$ & $3(0-6)$ & $2(0-6)$ & $2(0-6)$ & - \\
\hline
\end{tabular}

Abbreviations: $\mathrm{N}=$ number, ECOG = Eastern Cooperative Oncology Group, PS = Performance status, NSCLC $=$ Non-small cell lung cancer 


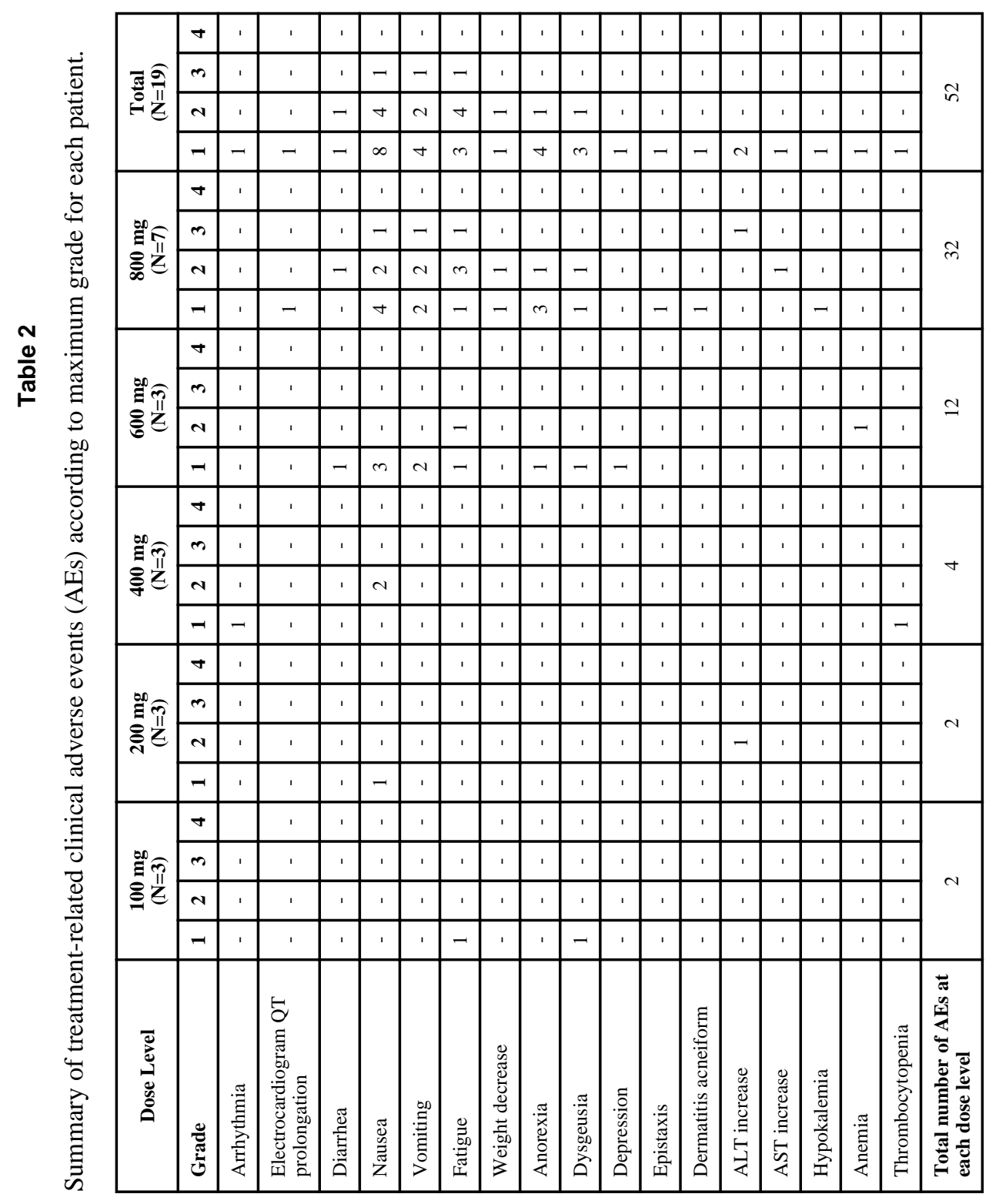

Clin Cancer Res. Author manuscript; available in PMC 2014 April 01. 
Table 3

Summary of pharmacokinetic parameters following single and multiple dosing of resminostat.

\begin{tabular}{|c|c|c|c|c|c|}
\hline \multicolumn{6}{|c|}{ Resminostat $^{a}$ pharmacokinetic parameter values following single oral administration (day 1) } \\
\hline Dose & $100 \mathrm{mg}$ & $200 \mathrm{mg}$ & $400 \mathrm{mg}$ & $600 \mathrm{mg}$ & $800 \mathrm{mg}$ \\
\hline $\mathrm{N}$ of patients & 3 & 3 & 3 & 3 & 7 \\
\hline Half-life (h) & $\begin{array}{c}3.77 \\
(3.21-4.41)\end{array}$ & $\begin{array}{c}3.12 \\
(2.75-3.53)\end{array}$ & $\begin{array}{c}3.44 \\
(3.22-3.67)\end{array}$ & $\begin{array}{c}2.84 \\
(2.74-2.94)\end{array}$ & $\begin{array}{c}2.81 \\
(2.35-3.37)\end{array}$ \\
\hline Clearance $(\mathbf{m L} / \mathbf{h})$ & $\begin{array}{c}80900 \\
(51600,127000)\end{array}$ & $\begin{array}{c}53800 \\
(45000,64300)\end{array}$ & $\begin{array}{c}52500 \\
(50600,54400)\end{array}$ & $\begin{array}{c}41400 \\
(32200,53300)\end{array}$ & $\begin{array}{c}37700 \\
(29500,48200)\end{array}$ \\
\hline $\mathrm{C}_{\max }(\mathrm{ng} / \mathrm{mL})$ & $\begin{array}{c}487 \\
(331,716)\end{array}$ & $\begin{array}{c}1470 \\
(915,2370)\end{array}$ & $\begin{array}{c}2160 \\
(1720,2710)\end{array}$ & $\begin{array}{c}4100 \\
(3060,5480)\end{array}$ & $\begin{array}{c}4970 \\
(3540,6980)\end{array}$ \\
\hline $\mathrm{AUC}_{\mathrm{inf}}(\mathrm{h} \times \mathbf{n g} / \mathbf{m L})$ & $\begin{array}{c}1236 \\
(788,1936)\end{array}$ & $\begin{array}{c}3718 \\
(3110,4445)\end{array}$ & $\begin{array}{c}7626 \\
(7355,7906)\end{array}$ & $\begin{array}{c}14480 \\
(11260,18620)\end{array}$ & $\begin{array}{c}21200 \\
(16580,27090)\end{array}$ \\
\hline \multicolumn{6}{|c|}{ Resminostat $^{a}$ pharmacokinetic parameter values following multiple oral administration (day 5) } \\
\hline Dose & $100 \mathrm{mg}$ & $200 \mathrm{mg}$ & $400 \mathrm{mg}$ & $600 \mathrm{mg}$ & $800 \mathrm{mg}$ \\
\hline $\mathrm{N}$ of patients & 3 & 3 & 3 & 3 & 6 \\
\hline$t_{\max }(h)$ & $\begin{array}{c}2.00 \\
(0.75-2.50)\end{array}$ & $\begin{array}{c}2.00 \\
(1.50-5.00)\end{array}$ & $\begin{array}{c}2.50 \\
(1.50-4.00)\end{array}$ & $\begin{array}{c}2.50 \\
(1.50-4.00)\end{array}$ & $\begin{array}{c}1.75 \\
(1.50-6.00)\end{array}$ \\
\hline Clearance $(\mathrm{mL} / \mathrm{h})$ & $\begin{array}{c}75100 \\
(51000,110000)\end{array}$ & $\begin{array}{c}59500 \\
(45500,77800)\end{array}$ & $\begin{array}{c}49300 \\
(39900,61000)\end{array}$ & $\begin{array}{c}31100 \\
(27100,35600)^{b}\end{array}$ & $\begin{array}{c}41200 \\
(29900,56800)\end{array}$ \\
\hline $\mathrm{C}_{\max }(\mathrm{ng} / \mathrm{mL})$ & $\begin{array}{c}373 \\
(222,627)\end{array}$ & $\begin{array}{c}1060 \\
(536,2080)\end{array}$ & $\begin{array}{c}2270 \\
(1650,3130)\end{array}$ & $\begin{array}{c}3000 \\
(1640,5460)\end{array}$ & $\begin{array}{c}4510 \\
(2280,8940)\end{array}$ \\
\hline $\mathbf{A U C}_{\mathrm{ss}}(\mathbf{h} \times \mathbf{n g} / \mathbf{m L})$ & $\begin{array}{c}1332 \\
(905.1,1961)\end{array}$ & $\begin{array}{c}3362 \\
(2572,4395)\end{array}$ & $\begin{array}{c}8108 \\
(6559,10020)\end{array}$ & $\begin{array}{c}19310 \\
(16860,22110)^{b}\end{array}$ & $\begin{array}{c}19430 \\
(14100,26780)\end{array}$ \\
\hline
\end{tabular}

Abbreviations: $t_{\max }=$ time of peak plasma concentration, $\mathrm{C}_{\max }=$ peak plasma concentration, $\mathrm{AUC} \mathrm{SS}_{\mathrm{S}}=$ area under the curve for steady state levels

Geometric means (68\% range) have been provided except for $t_{\max }$ where median values $(\min / \max )$ have been presented instead.

$a_{\text {free-base }}$

$b_{\mathrm{n}=2}$ 